\title{
THE STAR COMPACTIFICATION
}

\author{
G.D. RICHARDSON \\ Department of Mathematics \\ East Carolina University \\ Greenville, N. C. 27834 \\ D.C. KENT \\ Department of Pure and Applied Mathematics \\ Washington State University \\ Pullman, Washington 99164
}

(Received September 18, 1980)

ABSTRACT. The relationships between a convergence space and its star compactification is studied. Special attention is given to lifting properties of this compactification. In particular, it is shown that a natural extension of any continuous function to the respective compactification spaces is $\theta$-continuous. KEY WORDS AND PHRASES. Convergence space, compactification, G-space, R-series, natural extension, $\theta$-continuous function, proper map, open map. 


\section{INTRODUCTION.}

We study a convergence space compactification which was introduced by one of the authors in 1970 (see [11]). The star compactification $k^{*}=\left(X^{*}, 1^{*}\right)$ of a convergence space $X$ is constructed by adjoining to $X$ the set $X^{\prime}$ of all non-convergent ultrafilters on $\mathrm{X}$ and constructing a compact convergence structure on $\mathrm{X}^{*}=\mathrm{X} \cup \mathrm{X}^{\prime}$ in a natural way. It is proved in [11] that a continuous function from a $T_{2}$ space $X$ into a compact $T_{3}$ space $Y$ has a continuous extension to $X^{*}$. The authors published a survey paper, [7], concerning the existence of largest and smallest convergence space compactifications relative to various constraints. In all cases studied, the largest compactification, whenever it existed, turned out to be $k^{*}$. In a more recent paper, [9], we showed that $k^{*}$ can be used to characterize $\omega$-regular and completely regular spaces. These results suggest that further investigation of the star compactification is appropriate.

In Section 2, we examine the relationship between the decomposition series of $\mathrm{X}$ and $\mathrm{X}^{*}$, showing that the lengths of these series can differ by at most one. These results yield a method for constructing compact $T_{2}$ spaces with arbitrarily long decomposition series. In Section 3 , the $\mathrm{R}$-series of $\mathrm{X}$ and $\mathrm{X}^{*}$ are compared. By means of the R-series, the notion of $\theta$-continuity and other $\theta$-mapping properties (see [2], [3]) are extended to convergence spaces.

If $\mathrm{f}$ is a function from a space $\mathrm{X}$ to a space $\mathrm{Y}$, then a "natural extension" $f_{*}: X^{*} \rightarrow Y^{*}$ is defined in Section 4. The natural extension is unique if $Y$ is $\mathrm{T}_{2}$ and coincides with the continuous extension constructed in [11] when $\mathrm{Y}$ is compact and $T_{3}$. The main result of Section 4 is that any natural extension $f_{*}$ is $\theta$-continuous whenever $f$ is continuous. This result is used to obtain, among other things, an alternate construction of $\beta X$ for a Tychonoff topological space $X$. Section 5 examines conditions on $f, X$, and $Y$ under which $f_{*}$ is continuous, and Section 6 gives conditions under which $f_{*}$ preserves certain quotient-type mapping 
properties, such as "open", "proper", and "perfect".

\section{DECOMPOSITION SERIES.}

The reader is asked to refer to [7] for convergence space notation and terminology not given here, as well as additional information about the star compactification. As in [7], space will always mean convergence space, and the abbreviation "u.f." is often used for "ultrafilter". The separation axioms $T_{1}$ (singletons are closed), $\mathrm{T}_{2}$ (convergent filters have unique limits), and $\mathrm{T}_{3}$ (regular plus $\mathrm{T}_{2}$ ) will be used, but no separation axioms are assumed unless such is explicitly stated.

Given a space $X$, let $F(X)$ (resp. $U(X)$ ) be the set of all filters (resp., ultrafilters) on $X$. Let $X^{\prime}$ be the set of all non-convergent members of $U(X)$, and $X^{*}=X \cup X^{\prime}$. If $A \subseteq X$, define $A^{\prime}=\left\{F \in X^{\prime}: A \in\right\}$, and $A^{*}=A \cup A^{\prime}$. If $\mathfrak{F} \in \mathrm{F}(\mathrm{X})$, and $\mathrm{F}^{\prime} \neq \phi$ for all $\mathrm{F} \in \mathcal{F}$, then let $\mathfrak{F}^{\prime \prime}$ be the filter generated by

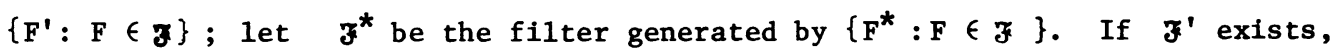
then $3^{*}=z^{*} \cap z^{\prime}$; otherwise, $z^{*}=z^{*}$. We omit the easy proofs of the first two propositions.

PROPOSITION 2.1. The following equalities hold for any subsets $A, B$ of $X$ : $A^{\prime} \cup B^{\prime}=(A \cup B)^{\prime} ; A^{\prime} \cap B^{\prime}=(A \cap B)^{\prime} ; A^{*} \cup B^{*}=(A \cup B)^{*} ; A^{*} \cap B^{*}=(A \cap B)^{*}$. Let $X$ be a space, $a \in F\left(X^{*}\right)$, and $X^{\prime} \in G$. Define $a^{\wedge}$ to be the filter on $X$ generated by $\left\{A \subseteq X: A^{\prime} \in G\right\}$.

PROPOSITION 2.2. (a) If $3 \in \mathrm{F}(\mathrm{X}), \& \in \mathrm{F}\left(\mathrm{X}^{*}\right)$, and $\& \geq 3^{*}$, then $\&^{\wedge} \geq z^{*}$.

(b) If $z^{\prime} \in(x)$ and $z^{\prime}$ exists, then $\left(z^{\prime}\right)^{\wedge}=z^{x}$.

(c) If $a \in u\left(x^{*}\right)$ and $x^{\prime} \in a$, then $a^{\wedge} \in \mathcal{u}(x)$.

A convergence structure is defined on $x^{*}$ as follows:

For $x \in x, a+x$ in $x^{*}$ iff there is $3 \rightarrow x$ in $x$ such that $a \geq z^{*}$; for $\& \in X^{\prime}, a \rightarrow \&$ iff $a \geq \&^{*}$. Let $i^{*}$ denote the identity embedding of $x$ into $\mathrm{X}^{*}$; it is proved in [11] that $\kappa^{*}=\left(\mathrm{X}^{*}, \mathrm{i}^{*}\right)$ is a compactification of $\mathrm{X}$ which is $\mathrm{T}_{2}$ 
whenever $X$ is $T_{2}$. It is immediate from the construction that, for any noncompact space $\mathrm{X}, \mathrm{X}^{*}-\mathrm{X}$ is a $\mathrm{T}_{2}$ pretopological space; thus $\mathrm{X}^{*}$ is pretopological whenever $X$ is pretopological. The universal property of $K^{*}$ established in [11] will be obtained in Section 3 as a corollary of a much more general result.

A subset $A$ of space $X$ is bounded if each ultrafilter containing $A$ is convergent. $X$ is said to be locally bounded if each convergent filter contains a bounded set. $X$ is essentially bounded if $F \in X^{\prime}$ implies that the filters

$J$ and $\cap\left\{\& \in X^{\prime}: \& \neq Z\right\}$ contain disjoint sets. The next proposition is proved in [7].

PROPOSITION 2.3. (a) $X$ is locally bounded iff $X$ is open in $X^{*}$.

(b) $X$ is essentially bounded iff $x^{*}-X$ is discrete.

We shall next consider the relationship between the closure operators of $x$ and $\mathrm{x}^{*}$. Let $\mathrm{cl}_{\mathrm{X}}$ be the closure operator on a space $\mathrm{X}$. For an ordinal number $\alpha$, we define:

$\mathrm{Cl}_{\mathbf{X}}^{\circ} \mathbf{A}=\mathbf{A}$

${ }^{c 1}{ }_{X}^{1} A={ }^{c 1} X$

$\mathrm{c1}_{\mathrm{X}}^{\alpha} \mathrm{A}=\mathrm{c1}_{\mathrm{X}}\left(\mathrm{c} 1_{\mathrm{X}}^{\alpha-1} \mathrm{~A}\right)$ if $\alpha-1$ exists

$c 1_{X}^{\alpha} A=\underset{\beta<\alpha}{U} c 1_{X}^{\beta} A$, if $\alpha$ is a limit ordinal.

The smallest ordinal $\alpha$ such that $\mathrm{cl}_{\mathrm{X}}^{\alpha} \mathrm{A}=\mathrm{c1}{ }_{\mathrm{X}}^{\alpha+1} \mathrm{~A}$ for all $\mathrm{A} \subseteq \mathrm{X}$ is called the length of the decomposition series of $X$ and denoted by $l_{D}(X)$. The relationship between $\ell_{D}(X)$ and $\ell_{D}\left(X^{*}\right)$ can be obtained with the help of several lemmas.

For the remainder of this section, we shall assume that $\mathrm{X}$ is an arbitrary space; $\left(\mathrm{X}^{*}, \mathrm{I}^{*}\right)$ will always denote the star compactification of $\mathrm{X}$. Let $\omega$ be the smallest infinite ordinal number.

LEMMA 2.4. If $A \subseteq X$, then $c 1_{X}^{n} A=c 1_{X}^{n} A \cup\left(c 1_{X}^{n-1} A\right)^{\prime}$. 
PROOF. It suffices to prove this result for $n=2$. Note that $\mathrm{c1}_{\mathrm{X}}^{2} \mathrm{~A} \cup\left(\mathrm{c1} 1_{\mathrm{X}} \mathrm{A}\right)^{\prime} \subseteq \mathrm{cl}_{\mathrm{X}}^{2} * \mathrm{~A}$ is obvious. If $\mathrm{x} \in\left(\mathrm{cl}_{\mathrm{X}^{*}}^{2} A\right) \cap \mathrm{X}$, then there is $a \in U\left(x^{*}\right)$ such that $a \geq \&^{*}$, and $x \in a$ implies $\left.a\right|_{X} \geq \&$. Thus $x \in \mathrm{cl}_{X}^{2} A$. If $z \in \mathrm{cl}_{X^{*}}^{2} A \cap X^{\prime}$, then it is easy to see that $c 1_{X} A \in J$, and so $z \in\left(c 1_{X} A\right)^{\prime}$.

We next describe ${ }^{c 1} X^{*} B$ for $B \subseteq X^{\prime}$. For this purpose, it is necessary to introduce some additional terminology. If $B \subseteq X^{\prime}$, let ${ }_{B}=\cap\{\mathbb{F} \in B\}$; note that $\sharp_{B} \in F(X)$. Define $B^{2}=\left\{\& \in X^{\prime}: \& \geq H_{B}\right\}$, and $B^{2}=\{x \in X: \exists \exists \in U(X)$ such that $F \rightarrow x$ in $X$ and $\left.F \geq H_{B}\right\}$. Note that $\left(B^{\sim}\right)^{\sim}=B^{\sim}$ and $\left(B^{\sim}\right)^{\sim}=B^{\nu}$.

LEMMA 2.5. If $B \subseteq X^{\prime}$, then $\mathrm{cl}_{X^{*}}^{n} B=B^{\sim} U\left(c_{X}^{n-1} B^{\vee}\right) \cup\left(c_{X}^{n-2} B^{\nu}\right)^{\prime}$.

PROOF. For $n=1$, the statement becomes $\mathrm{cl}_{X} * B^{2}=B^{\sim} \cup B^{2}$. If $\& \in\left(c 1_{X^{*}} B\right) \cap X^{\prime}$, then there is $a \in U\left(X^{*}\right)$ such that $a \rightarrow \&$ in $x^{*}$ and $B \in a$. Thus $a \geq \&^{\prime}$, and so $B \cap G^{\prime} \neq \phi$ for all $G \in \&$. This implies $\& \geq A_{B}$, and so $\& \in B^{\sim}$. If $x \in\left(c 1_{X} B\right) \cap x$, then there is an u.f. $G$ containing $B$ and $a$ filter $\rightarrow x$ such that $a \geq z^{\prime}$. By Proposition 2.2, $a^{\wedge} \geq \mathcal{F}^{\prime}$, and so $a^{\wedge} \rightarrow x$ in $x$. Also, $a^{\wedge} \in U(x)$ and $a^{\wedge} \geq\left(a^{\wedge}\right)^{\prime}$. Letting $\&=a^{\wedge}$, we have $B \cap G^{\prime} \neq \phi$ for all $G \in \&$. Thus $\& \geq H_{B}$, and $x \in B^{2}$.

Conversely, if $x \in B^{2}$, then there is $z \in U(x)$ such that $z \rightarrow x$ and $z \geq H_{B}$. For each $F \in z$, choose $\&_{F} \in B$ such that $F \in \&_{F}$. Let $a$ be the filter of sections of the net $\left(\&_{F}\right)_{F \in J^{*}}$ Then $a \geq 3^{*}$ implies $G \rightarrow x$ in $x^{*}$, and so $x \in \mathrm{cl}_{\mathrm{X}} * \mathrm{~B}$. A similar argument shows that $\mathrm{B}^{\sim} \subseteq \mathrm{Cl}_{\mathrm{X}} \mathrm{B}^{\mathrm{B}}$. This establishes the result for $n=1$.

If $n=2$, then $\mathrm{cl}_{X^{*}}^{2} B=c 1_{X^{*}}\left(B^{\sim} U B^{\sim}\right)=\left(B^{\sim}\right)^{\sim} U\left(B^{\sim}\right)^{\sim} U\left(c 1_{X} B^{\sim}\right)^{\prime}$ follows with the help of Lemma 2.4. By the remarks preceding Lemma $2.5,\left(B^{\sim}\right)^{\sim}=B^{\sim}$ and $\left(B^{2}\right)^{\sim}=B^{\nu}=B^{\nu} \subseteq c X^{2}$. This establishes the result for $n=2$. The generalization to arbitrary $\mathrm{n}$ is now a trivial induction argument.

COROLLARY 2.6. If $A \subseteq X$, then $\mathrm{cl}_{X^{*}} A=c 1_{X} A^{*}$. 
PROOF. $c 1_{X^{*}} A^{*}=c 1_{X^{*}} A \cup{ }^{c} x^{*} A^{\prime}$. By Lemma 2.5, $c 1_{X^{*}} A^{\prime}=\left(A^{\prime}\right)^{\sim} U\left(A^{\prime}\right)^{\sim}$. It is easy to check that $\left(A^{\prime}\right)^{\sim}=A^{\prime} \subseteq c^{c 1} x^{* A}$, and $\left(A^{\prime}\right)^{\vee} \subseteq{ }^{c 1} x^{A} \subseteq c x^{*} A$.

LEMMA 2.7 (a) If $A \subseteq X$ and $\alpha$ is a non-limit ordinal, then $\mathrm{cl}_{X^{*}}^{\alpha} A=\mathrm{cl}_{X}^{\alpha} A \cup\left(c 1_{X}^{\alpha-1} A\right)^{\prime}$.

(b) If $\alpha$ is a limit ordinal, then $\mathrm{cl}_{X^{*} A}^{\alpha}=\mathrm{cl}_{X}^{\alpha} A \cup\left(U\left\{\left(c 1_{X}^{\beta} A\right)^{\prime}: B<\alpha\right\}\right)$.

PROOF. Using transfinite induction along with Lemma 2.4 , the results follow easily from both limit and non-1imit ordinals, except for the case $\alpha=\gamma+1$, where $\gamma$ is a limit ordinal. In this case we have

$$
\mathrm{cl}_{X^{*}}^{\gamma+1} A={ }^{*} 1_{X} *\left(c_{X}^{\gamma} A \cup B\right)=c_{X}^{\gamma+1} A \cup\left(c_{X}^{\gamma} A\right)^{\prime} \cup B^{\sim} \cup B^{\sim},
$$

where $B=U\left\{\left(c_{X}^{\beta} A\right)^{\prime}: B<\alpha\right\}$. It is not difficult to show that $B^{2} \subseteq c_{X}^{\gamma+1} A$ and $B^{\sim} \subseteq\left(\mathrm{cl}_{X}^{\gamma} A\right)^{\prime}$; we omit the details.

The symbol $\lambda \mathrm{X}$ represents the topological modification of $\mathrm{x}$, $1 . e .$, the finest topological space on $\mathrm{X}$ coarser than $\mathrm{X}$.

THEOREM 2.8. $\lambda \mathrm{X}$ is a subspace of $\lambda \mathrm{x}^{*}$.

PROOF. Let $X_{1}=\left.\lambda X^{*}\right|_{X}$. Then $\lambda X \geq X_{1}$ is clear. Let $A \subseteq X$ be $\lambda X$-closed. Then $c 1_{X^{*}} A=c 1_{X} A \cup A^{\prime}=A \cup A^{\prime}=A^{*}=c 1_{X} A^{*}$, by Corollary 2.6. Thus $A^{*}$ is closed in $\lambda X^{*}$ and $A=A^{*} \cap X$, which implies $A$ is $X_{1}$-closed.

THEOREM 2.9. (a) If $1 \leq \ell_{D}(X)<\omega$, then $\ell_{D}(X) \leq \ell_{D}\left(X^{*}\right) \leq \ell_{D}(X)+1$.

(b) If $\ell_{D}(X) \geq w$, then $\ell_{D}(X)=\ell_{D}\left(X^{*}\right)$.

PROOF. Let $A \subseteq X^{*}$ and $A=B \cup C$, where $B=A \cap X, C=A \cap X^{\prime}$. Then, by Lemmas 2.4 and 2.5, $\mathrm{cl}_{X^{*}}^{\mathrm{n} A}=\mathrm{cl}_{X}^{\mathrm{n}} B \cup\left(\mathrm{cl}_{X}^{\mathrm{n}-1} B\right)^{\prime} U\left(\mathrm{cl}_{X}^{\mathrm{n}-1} \mathrm{c}^{\sim}\right) \cup\left(\mathrm{cl}_{X}^{\mathrm{n}-2} \mathrm{c}^{\sim}\right) \cup \mathrm{c}^{\sim}$. If $c 1_{X}^{k}$ is idempotent, it follows that $\mathrm{cl}_{\mathrm{X}^{*}}^{\mathrm{k}+1}$ must be idempotent. Thus $\ell_{D}\left(X^{*}\right) \leq \ell_{D}(X)+1$. It follows easily from Lemma 2.4 that $c l_{X}^{k}$ is idempotent if $\mathrm{cl}_{\mathrm{X}^{*}}^{\mathrm{k}}$ is idempotent. Thus (a) is established.

Statement (b) follows easily from Lemma 2.6.

COROLLARY 2.10. If $X$ is a topological space, then $\ell_{D}\left(X^{*}\right) \leq 2$. 
We define a $\mathrm{G}$-space to be a regular space with the property that $\mathrm{cl}_{\mathrm{X}} \mathrm{F}=\mathrm{F}$ for all $\in X^{\prime}$; this concept (but not the terminology) was introduced by Gazik [4]. Discrete spaces and compact regular spaces are the most obvious examples of G-spaces. Another class of G-spaces are the ultraspaces, which are the topological spaces having exactly one convergent ultrafilter. The next theorem is proved in [4] in the case where $X$ is $T_{2}$; removal of the $T_{2}$ assumption causes no problems in the proof.

THEOREM 2.11. $\mathrm{X}^{*}$ is regular iff $\mathrm{X}$ is a G-space.

A regular space is symmetric if $z \rightarrow x$ whenever $z \rightarrow y$ and $\dot{y} \rightarrow x$. Examples of symmetric spaces include $\mathrm{T}_{3}$ spaces, regular topological spaces, and regular convergence groups. It is shown in [14] that a compact symmetric space has the same ultrafilter convergence as a compact regular topological space.

PROPOSITION 2.12. If $X$ is a symmetric G-space, then $x^{*}$ is symmetric. PROOF. Let $Q \rightarrow \alpha$ and $\dot{\alpha} \rightarrow \beta$ in $x^{*}$. By the construction of $x^{*}$, we can conclude that both $\alpha$ and $\beta$ are in $x$. Since $a \rightarrow \alpha$, there is $z \rightarrow \alpha$ in $x$ such that $a \geq 3^{*}$. Since $x$ is symmetric, $x \rightarrow \beta$ in $x$, and so $a \rightarrow \beta$ in $x^{*}$.

THEOREM 2.13. (a) If $X$ is a G-space, then $\ell_{D}\left(X^{*}\right) \leq 2$ and $\ell_{D}(X) \leq 2$.

(b) If $X$ is a symetric G-space, then $\ell_{D}\left(X^{*}\right) \leq 1$.

(c) If $X$ is a $T_{2}$ topological space, then $l_{D}\left(X^{*}\right) \leq 1$ iff $X$ is a G-space. PROOF. (a) If $X$ is a G-space, then $X^{*}$ is a compact regular space by Theorem 2.11, and it follows by Theorem $2.4(a),[14]$, that $\ell_{D}\left(X^{*}\right) \leq 2$. The second inequality follows from the first and Theorem 2.9.

Statement (b) follows immediately from Proposition 2.12 and Theorem 2.4(b), [14].

(c) If $X$ is a topological G-space, then $X$ is symmetric, and so $l_{D}\left(X^{*}\right) \leq 1$ by (b). If $l_{D}\left(X^{*}\right) \leq 1$, then $X^{*}$ is a compact $T_{2}$ topological space, and hence $X^{*}$ is regular. By Theorem $2.11, \mathrm{X}$ is a G-space. 
It should be noted that $\ell_{D}(Y)=0$ iff $Y$ is discrete, and consequently $\ell_{D}\left(X^{*}\right)=0$ iff $X$ is a finite discrete space. If $X$ is not a finite discrete space, we can replace $" \ell_{D}\left(X^{*}\right) \leq 1$ "by $\ell_{D}\left(X^{*}\right)=1$ " in $(b)$ and $(c)$ of Theorem 2.13.

\section{R-SERIES.}

In this section, we summarize some results on the R-series of a space (originally studied in [13] and [14]), obtain a few results on the R-series of $x^{*}$, and lay the groundwork for many of the results of Section 4 .

Starting with a space $x$, an ordinal family of spaces $\left\{r_{\alpha} x\right\}$ is defined on the same underlying set as follows: $r_{0}(X)=X$

$z \rightarrow x$ in $r_{1} X$ iff there exist $n \in N$ and $\& \rightarrow x$ in $x$ such that $z \geq c 1_{X}^{n} \&$ $z \rightarrow x$ in $r_{\alpha} X$ iff there exist $n \in N, \& \rightarrow x$ in $X$ and $\beta<\alpha$ such that $z \geq c 1_{r_{B} X}^{n}$. The family $\left\{r_{\alpha} x\right\}$ is called the $\underline{R-s e r i e s}$ of $x$. If $\gamma$ is the least ordinal such that $r_{\gamma} X=r_{\gamma+1} X$, then $r_{\gamma} X$ is denoted $x_{r}$, and $\gamma$ is called the length of the $\underline{R-s e r i e s}$ of $X$ and denoted by $\ell_{R}(X)$. Note that $X$ is regular iff $\ell_{R}(X)=0$. It is shown in [13] that $x_{r}$ is the finest regular space coarser than $X$, and $x_{r}$ is called the regular modification of $\mathrm{x}$.

of course, $X_{r}$ will not in general be $T_{2}$, even when $X$ is $T_{2}$. A $T_{3}$ space associated with $X$ (we use $T_{3}$ to mean regular plus $T_{1}$ ) is constructed as follows. First, define an equivalence relation among the elements of $x$ by $: x \sim y$ if $\dot{x} \rightarrow y$ in $\mathrm{X}_{\mathrm{r}}$. Let $\mathrm{X}_{\mathrm{s}}$ be the set of equivalence classes with the quotient convergence structure derived from $x_{r}$.

If $\mathrm{f}: \mathrm{X} \rightarrow \mathrm{Y}$, let $\overline{\mathrm{f}}: \mathrm{X}_{\mathrm{S}} \rightarrow \mathrm{Y}_{\mathrm{S}}$ be the (unique) function which makes the following diagram commute:

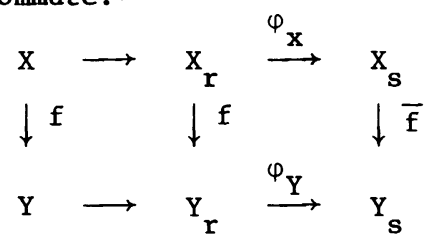


where the maps from $X$ to $X_{r}$ and $Y$ to $Y_{r}$ are the respective identity maps, and $\varphi_{\mathrm{X}}, \varphi_{\mathrm{Y}}$ are the respective quotient maps.

Let $\sigma \mathrm{X}$ denote the symmetric modification of $\mathrm{X}$, i.e., the finest symmetric space coarser than $X$. The next proposition follows immediately from results of [13] and [14].

PROPOSITION 3.1. If $\mathrm{f}: \mathrm{X} \rightarrow \mathrm{Y}$ is continuous, then each map in the following commutative diagram (in which all non-labeled vertical maps are $f$ and all nonlabeled horizontal maps are identities) is continuous.

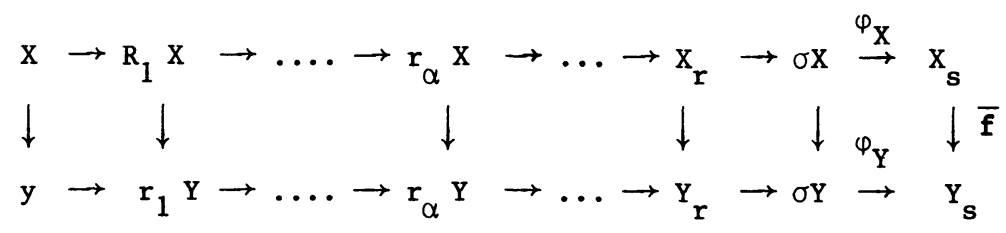

PROPOSITION 3.2. For any space $X, r_{1} X$ is a subspace of $r_{1} X^{*}$.

PROOF. Let $X_{1}$ be the restriction of $r_{1} x^{*}$ to $x$. Since $i^{*}: x \rightarrow x^{*}$ is continuous, it follows from Proposition 3.1 that $i^{*}: r_{1} X \rightarrow r_{1} X^{*}$ is continuous, and thus $r_{1} x \geq x_{1}$. On the other hand, let $3 \rightarrow x$ in $x_{1}$. Then there is $a \rightarrow x$ in $x^{*}$ and $n \in N$ such that $z \geq{ }^{2} x^{n} G$. Since $G \rightarrow x$ in $x^{*}$, there is $\& \rightarrow x$ in $X$ such that $a \geq \&^{*}$, and so we have $3 \geq \mathrm{cl}_{X^{*}}^{n} \&^{*}=\mathrm{cl}_{X^{*}}^{n} \&=\mathrm{cl}_{X}^{n} \& \cap\left(\mathrm{cl}_{X}^{n-1} \&^{\prime}\right)$, by Lemma 2.4 and Corollary 2.6. Since $J \in F(X), \quad \exists \geq c 1_{X}^{n} \&$, and so $\exists \rightarrow x$ in $\mathrm{r}_{1} \mathrm{X}$.

PROPOSITION 3.3. If $X$ is a locally compact $T_{3}$ space, then $r_{2} X$ is a subspace of $\mathrm{r}_{2} \mathrm{x}^{*}$.

PROOF. It is sufficient to show that for any $A \subseteq X, c^{n} r_{1} x A=\left(c^{n} r_{1} X^{*} A\right) \cap X$ for all $n \in N$, and this will be proved by induction. For $n=1$, the equality follows by Proposition 3.2. If the equality holds for $n$ and $x \in\left(c 1_{r_{1} X^{*} A}^{n+1} \cap x\right.$, then there is $3 \rightarrow x$ and $k \in N$ such that $\left(c 1_{X^{*}}^{k} F\right) \cap\left(c 1_{\left.r_{1} X^{*} A\right) \neq \phi \text { for all }}^{n}\right.$ $F \in$. Since $X$ is locally compact and $T_{3}$, we may assume without loss of 
generality that 3 has a filter base of compact sets, and so $c 1_{X^{*}}^{k} 3=c 1_{X}^{k} 3$. From this observation, along with the induction hypothesis, we may conclude that $x \in \mathrm{cl}_{\mathbf{r}_{1} \mathrm{X}}^{\mathrm{n}+1} \mathrm{~A}$.

It is not true in general that $r_{\alpha} X$ is a subspace of $r_{\alpha} X^{*}$. To establish this fact, we need to make use of some theorems from [9].

A space $X$ is defined to be completely regular if it is a subspace of a symmetric compact space. It is shown in [9] that $X$ is completely regular iff it is a symmetric space with the same ultrafilter convergence as a completely regular topological space. Let $\omega \mathrm{X}$ denote the finest completely regular space coarser than $X$. A space $X$ is defined to be $\underline{\omega-r e g u l a r}$ if $3 \rightarrow x$ implies $c 1 \omega X \rightarrow x$. It is proved in [9] that $X$ is $\omega$-regular iff $X$ is a subspace of a compact regular space. The $\omega$-regular spaces include the completely regular spaces and also the c-embedded spaces of Binz [1].

PROPOSITION 3.4. (a) If $X$ is a regular space which is not $\omega$-regular, then $x_{r}$ is not a subspace of $X_{r}^{*}$, and $\ell_{R}\left(X^{*}\right) \geq 2$.

(b) If $X$ is a locally compact $T_{3}$ space which is not $\omega$-regular, then $\ell_{R}\left(X^{*}\right) \geq 3$.

PROOF. The first part of (a) follows from the aforementioned characterization of $\omega$-regular spaces as subspaces of compact regular spaces. The two statements concerning $l_{R}\left(X^{*}\right)$ follow by Proposition 3.2 and 3.3, respectively.

For any space $X$, let $C(X)$ be the set of all continuous real-valued functions on $\mathrm{X} . \quad \mathrm{A} \mathrm{T}_{3}$ topological space $\mathrm{X}$ for which $\mathrm{C}(\mathrm{X})$ consists only of constant functions is an example of a regular space which is not $\omega$-regular; for this space, $l_{R}(X)=0$ and $\ell_{R}\left(X^{*}\right) \geq 2$. An example of a regular space $X$ for which $\ell_{R}\left(X^{*}\right) \geq 3$ is obtained with the help of the following lemma.

LEMMA 3.5. A locally compact $T_{3}$ space $X$ is $\omega$-regular iff $C(X)$ separates points in $\mathrm{X}$. 
PROOF. If $X=\omega X$, then $\omega X$ is $T_{2}$ and so $C(X)$ separates points in $X$. Conversely, if $J \rightarrow x$ in $X$, then $J$ contains a compact set $A$. Since $C(X)$ separates points in $X, \omega X$ is $T_{2}$ and so the subspaces $\left.X\right|_{A}$ and $\left.\omega X\right|_{A}$ have the same ultrafilter convergence. It follows that ${ }^{c 1} X={ }^{c 1} \omega X \quad x \rightarrow x$ in $x$, and therefore $\mathrm{X}$ is $\omega$-regular.

EXAMPLE 3.6. Let $X$ be the set $[0,1]$. If $s$ is a sequence on the set $X$, let ${ }_{s}$ denote the filter generated by $s$ in the usual way. Let \& be the filter generated by the sequence $\left(\frac{1}{n}\right)$. Define a convergence on $X$ as follows:

(1) If $x \in\{0,1\}$, then $\rightarrow x$ iff there is a sequence $s$ converging to $x$ in the usual topology such that $3 \geq{ }_{s} ;(2) \mathfrak{J} \rightarrow 0$ iff there is a sequence $s$ converging to 0 in the usual topology, but not a subsequence of $\left(\frac{1}{n}\right)$, such that $z \geq z_{s} ;(3)=1$ iff $3 \geq z_{s}$, where $s$ is a sequence converging to 1 in the usual topology, or else $z \& \cap \dot{1}$. One may easily verify that the space $\mathrm{x}$ is locally compact and $T_{3}$, but $C(X)$ will not separate the points 0 and 1 . Thus, by Lemma 3.5, $\mathrm{X}$ is not $\omega$-regular, and it follows from Proposition 3.4 that $\ell_{R}\left(X^{*}\right) \geq 3$, whereas $\ell_{R}(X)=0$; this result contrasts with the conclusion of Theorem 2.9. One can also show (we omit the details) that $0 \rightarrow 1$ in $r_{3} x^{*}$. Since $r_{3} X=X$, it follows that $r_{3} X$ is not a subspace of $r_{3} X^{*}$. This shows that the conclusion of Proposition 3.3 cannot be improved without imposing additional conditions.

Gazik showed in [4] that a $\mathrm{T}_{3}$ pretopological G-space is a completely regular topological space. Another result along these lines is

PROPOSITION 3.7. (a) A symmetric G-space is completely regular.

(b) Every G-space' is $\omega$-regular.

PROOF. These statements follow immediately from Theorem 2.11, Proposition 2.12 , and the characterization of $\omega$-regular spaces obtained in [9]. 
THEOREM 3.8. Let $\mathrm{X}$ be a space.

(a) $X$ is regular iff $X$ is a subspace of $r_{1} X^{*}$.

(b) $X$ is w-regular iff $X$ is a subspace of $x_{r}^{*}$.

(c) $\mathrm{X}$ is completely regular iff $\mathrm{X}$ is a subspace of $\sigma \mathrm{X}^{*}$.

Proof. Statement (a) follows immediately from Proposition 3.2. Statements (b) and (c) are proved in [9].

For topological spaces $X$ and $Y$, function $f: X \rightarrow Y$ is defined to be $\underline{\theta \text {-continuous }}$ if, for every $x \in X$ and every neighborhood $v$ of $f(x)$, there is a neighborhood $u$ of $f(x)$ such that $f\left(c 1_{X} u\right) \subseteq c_{Y} v$.

PROPOSITION 3.9. Let $\mathrm{f}: \mathrm{X} \rightarrow \mathrm{Y}$, where $\mathrm{X}$ and $\mathrm{Y}$ are topological spaces. Then $\mathrm{f}: \mathrm{X} \rightarrow \mathrm{Y}$ is $\theta$-continuous iff $\mathrm{f}: \mathrm{r}_{1} \mathrm{X} \rightarrow \mathrm{r}_{1} \mathrm{Y}$ is continuous.

PROOF. Let $\mathrm{f}: \mathrm{X} \rightarrow \mathrm{Y}$ be $\theta$-continuous, and let $\rightarrow \mathrm{x}$ in $\mathrm{r}_{1} \mathrm{X}$. Then there is $\& \rightarrow x$ in $x$ such that $z \geq{ }^{c 1_{X}} \& \geq{ }^{c 1} x v(x)$, where $v(x)$ is the neighborhood filter at $x$. By $\theta$-continuity, $f\left(c 1_{X} v(x) \geq c 1_{Y} v(f(x))\right.$, and so $f(\pi) \geq c 1_{Y} v(f(x))$. The latter filter $r_{1} Y$-converges to $f(x)$, and so $\mathrm{f}: \mathrm{r}_{1} \mathrm{X} \rightarrow \mathrm{r}_{1} \mathrm{Y}$ is continuous. For the converse argument, one easily see that $f: r_{1} X \rightarrow r_{1} Y$ implies $f\left(c 1_{X} v(x)\right) \geq c 1_{Y} v(f(x))$ for all $x \in X$, which is equivalent to $\theta$-continuity of $\mathrm{f}: \mathrm{X} \rightarrow \mathrm{Y}$.

The characterization of $\theta$-continuity given in Proposition 3.9 is not suitable for a purely topological investigation, since $r_{1} X$ may fail to be topological even when $\mathrm{X}$ is topological. Perhaps this suggests that convergence spaces are the natural realm for the study of $\theta$-continuity. But in any event, we shall define a function $\mathrm{f}: \mathrm{X} \rightarrow \mathrm{Y}$ between arbitrary convergence spaces to be $\underline{\theta \text {-continuous }}$ if $\mathrm{f}: \mathrm{r}_{1} \mathrm{X} \rightarrow \mathrm{r}_{1} \mathrm{Y}$ is continuous.

More generally, if $P$ is any function property, then $f: X \rightarrow Y$ is defined to have property $\underline{\theta-\mathrm{P}}$ if $\mathrm{f}: \mathrm{r}_{1} \mathrm{X} \rightarrow \mathrm{r}_{1} \mathrm{Y}$ has property $\mathrm{P}$. Thus, one can speak of $\theta$-open maps, $\theta$-quotient maps, etc. Some of these " $\theta$-properties" will be studied 
briefly in Section 6 .

\section{NATURAL EXTENSIONS.}

Let $\mathrm{X}$ and $\mathrm{Y}$ be spaces and consider a function $\mathrm{f}: \mathrm{X} \rightarrow \mathrm{Y}$. A function $f_{*}: X^{*} \rightarrow Y^{*}$ is called a natural extension of $f$ if the following conditions are satisfied :

(1) $\left.f_{*}\right|_{X}=f$

(2) If $X^{\prime} \in X^{\prime}$ and $f(y)$ is convergent in $Y$, then $f_{*}(\xi)$ is an element of $Y$ to which $f(z)$ converges.

(3) If $z \in X^{\prime}$ and $f(z) \in Y^{\prime}$, then $f_{*}\left(z^{\prime}\right)=f(z) \in Y^{\prime}$.

If $\exists^{\prime} \in X^{\prime}$ implies $f(\exists) \in Y^{\prime}$, then $f$ is said to be weakly proper. If $\mathrm{f}: \mathrm{X} \rightarrow \mathrm{Y}$ is a weakly proper function, or if $\mathrm{Y}$ is $\mathrm{T}_{2}$, then the natural extension $f_{*}$ is unique; in general, $f$ may have many natural extensions. In the proposition and theorem that follow, when $f: X \rightarrow Y, f_{*}$ will be assumed to be an arbitrary natural extension of $f$.

The proof of the next lemma is straightforward and will be omitted.

LEMMA 4.1. If $\mathrm{f}: \mathrm{X} \rightarrow \mathrm{Y}$ and $\mathrm{A} \subseteq \mathrm{X}$, then :

(a) If $f$ is continuous, then $(f(A))^{*} \subseteq f_{*}\left(A^{*}\right) \subseteq f_{*}\left(c 1_{X^{*}} A\right) \subseteq c 1_{Y} f(A)$;

(b) If $f$ is continuous and $Y$ is $T_{2}$, then $f_{*}\left(c 1_{X^{*}} A\right)=c 1_{Y^{*}} f(A)$;

(c) If $f$ is weakly proper, then $f_{*}\left(A^{*}\right) \subseteq(f(A))^{*}$.

THEOREM 4.2. If $\mathrm{f}: \mathrm{X} \rightarrow \mathrm{Y}$ is continuous, then $\mathrm{f}_{*}: \mathrm{X}^{*} \rightarrow \mathrm{Y}^{*}$ is $\theta$-continuous.

PROOF. It is sufficient to show that, for each $z \in F(X), f_{*}\left(\mathrm{cl}_{X^{*}}^{n} z^{*}\right) \geq$ $c 1_{Y^{*}}^{n} f(\xi)$. If $F \in J$, then $c 1_{X^{*}}^{n} F=e 1_{X^{*}}^{n} F^{*}=c 1_{X}^{n} F \cup\left(c 1_{X}^{n-1} F\right)^{\prime}$ by Lemma 2.4 and Corollary 2.6. By continuity of $f, f_{\star}\left(c 1_{X}^{n} F\right)=f\left(c \cdot 1_{X}^{n} F\right) \subseteq c 1_{Y}^{n} f(F)$, and $f_{*}\left(c 1_{X}^{n-1} F\right)^{*} \subseteq{ }^{c 1} Y^{*} f\left(c 1_{X}^{n-1} F\right) \subseteq c 1_{Y} *\left(c 1_{Y}^{n-1} f(F)\right) \subseteq c 1_{Y}^{n} * f(F)$ follows with the help of Lemma 4.1. Thus $f_{*}\left(c 1_{X^{*}}^{n} F^{*}\right) \subseteq c 1_{Y^{*}}^{n} f(F)$, and the theorem is proved. 
COROLLARY 4.3. If $\mathrm{f}: \mathrm{X} \rightarrow \mathrm{Y}$ is continuous, then each map in the following commutative diagram (in which all non-labeled vertical maps are $f_{*}$ and all nonlabeled horizontal maps are identities) is continuous.

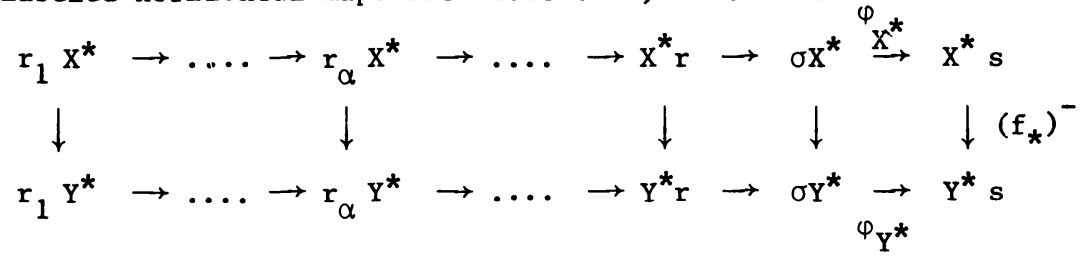

The next resul.t closely resembles, but is more general than, the extension property of the star compactification obtained in [11].

COROLIARY 4.4. If $\mathrm{f}: \mathrm{X} \rightarrow \mathrm{Y}$ is continuous and $\mathrm{Y}$ is compact and regular, then $f_{*}: X^{*} \rightarrow Y$ is continuous. If $Y$ is also $T_{2}$, then the extension $f_{\star}$ is unique.

PROOF. Under the given assumptions, $Y=Y^{*}=r_{1} Y^{*}$; since $X^{*} \geq r_{1} X^{*}$, the first statement is established. The second follows from an earlier remark.

In the next section we shall see that continuity of $\mathrm{f}: \mathrm{X} \rightarrow \mathrm{Y}$ does not guarantee the continuity of $\mathrm{f}_{*}: \mathrm{X}^{*} \rightarrow \mathrm{Y}^{*}$. If $\mathrm{X}$ is a regular space, let $X^{\sim}=r_{1} X^{*}$; then by Proposition $3.2 k^{\sim}=\left(X^{\sim}, i^{*}\right)$ is a compactification of $X$. Our study of the compactification $\kappa^{\sim}$ will be limited to the following proposition. PROPOSITION 4.5. Let $X$ and $Y$ be regular spaces.

(a) If $\mathrm{f}: \mathrm{X} \rightarrow \mathrm{Y}$ is continuous, then $\mathrm{f}_{*}: \mathrm{X}^{\sim} \rightarrow \mathrm{Y}^{\sim}$ is continuous.

(b) $\mathrm{X}^{\sim}$ is $T_{2}$ iff $\mathrm{X}$ is a $T_{2}$ G-space.

PROOF. Statement (a) follows immediately from Theorem 4.2.

(b) If $\mathrm{X}$ is a $T_{2}$ G-space, then $\mathrm{X}^{*}$ is regular by Proposition 2.10 , and so $\mathrm{X}^{\sim}=\mathrm{X}^{*}$ is $T_{2}$. If $X$ is not a G-sprace, then there is $z \in X^{\prime}$ such that $z>c c_{X}$, where $\& \in U(X)$ and $\& \neq \exists$. If $\& \stackrel{x}{\rightarrow} x$, then $\dot{x} \rightarrow \exists$ in $x^{\sim}$. If $\& \in X^{\prime}$, then the filter $\&_{1}$ on $x^{*}$ generated by \& converges in $x^{*}$ to both $\&$ and $z$.

It is shown in [12] that every completely regular $\mathrm{T}_{2}$ space has a Stone-Čech compactification. This compactification is regular and $\mathrm{T}_{2}$, has the universal property relative to the class of completely regular spaces, and agrees with the 
COROLLARY 4.7. If $X$ is a completely regular $T_{2}$ space, then $\left(X_{s}^{*}, 1_{*}^{-}\right)$is the Stone - Čech compactification of $X$.

If $\mathrm{X}$ is a Tychonoff topological space, then Corollary 4.7 gives a new method for constructing $\beta X$. Indeed, $\beta X$ is in this case the pretopological modification of $X^{*}$.

\section{CONTINUITY OF NATURAL EXTENSIONS.}

We next consider conditions under which a natural extension $f_{*}$ of a continuous function $f$ is continuous. For this purpose, we use some additional notation and terminology.

Let $\mathrm{f}: \mathrm{X} \rightarrow \mathrm{Y}$ be a continuous function, and let $\mathrm{f}_{\star}: \mathrm{X}^{\star} \rightarrow \mathrm{Y}^{\star}$ be a natural extension of $f$. For $A \subseteq X$, define $A_{f}^{\prime}=\left\{\xi \in A^{\prime}: f(\mathcal{F})\right.$ converges in $\left.Y\right\}$. Let $\Gamma_{f_{*}}(A)=f(A) \cup f_{*}\left(A_{f}^{\prime}\right)$; note that $\Gamma_{f_{*}}(A)=f_{*}\left(A^{*}\right) \cap Y$. If $z \in F(X)$ define $\Gamma_{f_{\star}}(\mathfrak{F}) \in F(Y)$ to be the filter generated by $\left\{\Gamma_{f_{\star}}(F): F \in F\right\} ; \exists \in F(X)$ is said to be $f_{\star}$-closed if $\Gamma_{f_{\star}}(\mathfrak{F})=f(\mathfrak{F})$.

PROPOSITION 5.1. Let $\mathrm{f}$ be a continuous map.

(1) $f_{\star}$ is continuous at $x \in f^{-1}(Y)$ iff $x \rightarrow x$ in $X$ implies that $\Gamma_{f_{*}} z \rightarrow f(x)$ in $Y$.

(2) $f_{\star}$ is continuous at $z \in f_{*}^{-1}(Y) \cap X$ iff $\Gamma_{f_{\star}}(z) \rightarrow f_{\star} J$ in $Y$.

(3) $f_{\star}$ is continuous at $\xi \in f_{*}^{-1}\left(Y^{\prime}\right)$ iff 3 is $f_{\star}$-closed. PROOF. If $\exists \in F(X)$ then one can easily show that $\Gamma_{f_{\star}} \exists \geq\left(\Gamma_{f_{*}}(\xi)\right)^{*}$; (1) and (2) follow from these inequalities.

(3) If $f_{*}$ is continuous at $\xi_{*}^{-1}\left(Y^{\prime}\right)$, then $\Gamma_{f_{\star}} \geq f_{*}\left(z^{*}\right) \geq(f(\xi))^{*}$, and hence $\Gamma_{f_{\star}} \xi=f(\xi)$. Conversely, $f_{\star}\left(\xi^{*}\right) \geq\left(\Gamma_{f}\right)^{\star}=(f \exists)^{*} \rightarrow f_{\star}$ in $^{*}$, and thus $\mathrm{f}_{\star}$ is continuous at $\mathrm{f}_{*}^{-1}\left(\mathrm{Y}^{\prime}\right)$.

COROLLARY 5.2. Let $\mathrm{f}: \mathrm{X} \rightarrow \mathrm{Y}$ be continuous, and $\mathrm{Y}$ a regular space. Then

(1) $f_{\star}$ is continuous at all points of $f_{*}^{-1}(Y)$.

(2) $f_{\star}$ is continuous iff each $3 \in f_{\star}^{-1}\left(Y^{\prime}\right)$ is $f_{\star}$-closed. 
topological Stone-Čech compactification relative to ultrafilter convergence when $\mathrm{X}$ is topological. We shall now give an alternate construction of this compactification using $k^{*}$.

For any space $X, X_{s}^{*}$ is a compact, regular, $T_{2}$ space. However it is not generally true that $x_{s}$ is a subspace of $x_{s}^{*}$. Recall the notation $\sigma x$ for the symmetric modification of $\mathrm{X}$.

THEOREM 4.6. If $\mathrm{X}$ is a space such that $\sigma \mathrm{X}$ is a subspace of $\sigma \mathrm{X}^{*}$, then $\sigma \mathrm{X}$ is completely regular, $X_{s}$ is completely regular and $T_{2}$, and $\left(x_{s}^{*}, i_{*}^{-}\right)$is the StoneČech compactification of $\mathrm{x}_{\mathrm{s}}$.

PROOF. By assumption, $\sigma \mathrm{X}$ is a subspace of a compact symmetric space, and hence completely regular. $X_{S}$ is $T_{2}$ by construction. In the diagram that follows

$$
\begin{gathered}
\sigma \mathrm{x} \stackrel{\stackrel{i_{*}}{\rightarrow} \sigma \mathrm{x}^{*}}{\varphi_{\mathrm{X}} \downarrow} \underset{\mathrm{x}_{\mathrm{s}}}{\stackrel{\mathrm{i}_{\star}}{\rightarrow}} \mathrm{x}_{\mathrm{s}}^{\star}
\end{gathered}
$$

the maps $\varphi_{\mathrm{X}}$ and $\varphi_{\mathrm{X}^{*}}$ are strongly open (see Proposition 2.2, [14]). This means that if $\& \rightarrow \alpha$ in $X_{s}$ and $x \in \varphi_{X}^{-1}(\alpha)$, then there is a filter $₹$ on $\sigma \mathrm{X}$ such that $z \rightarrow \mathrm{x}$ in $\sigma \mathrm{X}$ and $\varphi_{\mathrm{X}}(\tilde{z})=\&$. Using this property and the fact that $\sigma \mathrm{X}$ and $\sigma \mathrm{X}^{*}$ are symmetric, one can easily show that $X_{s}$ is densely embedded in $x_{s}^{*}$.

If $\mathrm{Y}$ is a regular, compact, $T_{2}$ space and $f: X_{s} \rightarrow Y$ is continuous, then define $F: X \rightarrow Y$ by $F(x)=f([x])$, where $[x]$ is the equivalence class in $X_{S}$ defined by $x$. It is easy to check that $F: X \rightarrow Y$ is continuous, and so by Corollary 4.3, $\left(\mathrm{F}_{*}\right)^{-}: \mathrm{X}_{\mathrm{S}}^{*} \rightarrow \mathrm{Y}_{\mathrm{S}}^{{ }^{*}}=\mathrm{Y}$ is continuous. $\left(\mathrm{F}_{*}\right)^{-}$is clearly an extension of $f$, and this. extension is unique because $Y$ is Hausdorff. Thus by the uniqueness of the Stone-Čech compactification established in [12], it follows that this compactification is equivalent to $\left(x_{s}^{*}, i_{*}^{-}\right)$. 
(3) If $X$ is essentially bounded, then $f_{*}$ is continuous.

PROOF. Statements (1) and (2) follow immediately from Proposition 5.1 and the fact that $\mathrm{Y}$ is regular. The assumption that $\mathrm{X}$ is essentially bounded (see Section 2 for this definition) guarantees that each $\exists \in f_{\star}^{-1}\left(Y^{\prime}\right)$ is $f_{\star}-c l o s e d$. PROPOSITION 5.3. If $\mathbf{f}: X \rightarrow Y$ is continuous and weakly proper, then $f_{\star}$ is continuous.

PROOF. If $f$ is weakly proper and $A \subseteq X$, then $A_{f}^{\prime}=\phi$. Thus each filter $z \in F(X)$ is $f_{\star}$-closed. The conditions (1) and (3) of Proposition 5.1 for continuity of $f_{*}$ are thus satisfied, while condition (2) is satisfied vacuously.

COROLLARY 5.4. If $X$ is a closed subspace of $Y$ and $f: X \rightarrow Y$ is the identity embedding, then $f_{*}: X^{*} \rightarrow Y^{*}$ is also an embedding.

PROOF. Since $X$ is closed in $Y$, $f$ is weakly proper; thus $f_{\star}$ is continuous by Proposition 5.3. $f_{\star}$ is clearly one-to-one, and by Lemma $4.1, f_{\star}\left(\xi^{*}\right)=f\left(\xi^{*}\right.$ for all $\underset{F}{F(X)}$. From this equality, it follows easily that $f_{\star}$ is an embedding.

PROPOSITION 5.5. The following statements about a regular space $\mathrm{Y}$ are equivalent.

(a) $Y$ is a G-space.

(b) Every natural extension of every continuous function into $Y$ is continuous.

(c) If $\mathrm{Z}$ and $\mathrm{Y}$ have the same set, $\mathrm{Z}$ is discrete, and $\mathrm{f}: \mathrm{Z} \rightarrow \mathrm{Y}$ is the identity, then $f_{*}$ is continuous.

PROOF. (a) $\Rightarrow(\mathrm{b})$. If $\mathrm{f}: \mathrm{X} \rightarrow \mathrm{Y}$ is continuous and $\mathrm{Y}$ is a G-space, then $\mathrm{Y}^{*}$ is regular by Theorem 2.11 and so $f_{\star}: X^{*} \rightarrow Y^{*}$ is continuous by Corollary 4.4.

(b) $\Rightarrow$ (c). Obvious.

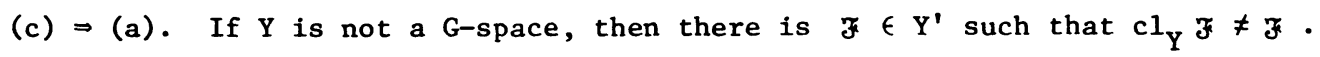
Since $Y$ is $T_{1}$ and $Z$ is discrete, it follows that $\Gamma_{f_{*}} \neq$ for some natural extension $f_{\star}$. Thus is not $f_{\star}$-closed, and by Proposition $5.1 f_{\star}$ is not 
continuous.

'The final result in this section is analogous to Proposition 5.1, but involves $\theta$-continuity rather than continuity. Since this result is of marginal interest, we shall omit the proof.

PROPOSITION 5.6. Let $\mathrm{f}: \mathrm{X} \rightarrow \mathrm{Y}$ be a $\theta$-continuous map.

(a) $f_{*}$ is $\theta$-continuous at each point $x \in X$.

(b) $f_{*}$ is $\theta$-continuous at $\xi^{*} f_{*}^{-1}(Y) \cap X^{\prime}$ iff $\left.f_{\left(c l^{n}\right.}^{n} X^{*}\right) \rightarrow f_{*}$ in $r_{1} Y$ for each $n \geq 1$.

(c) $f_{*}$ is $\theta$-continuous at $\mathcal{F}_{*}^{-1}\left(\mathrm{Y}^{\prime}\right)$ iff, for each $n \geq 1$, there is $m \geq 1$ such that $\mathrm{f}\left(\mathrm{cl}_{\mathrm{X}}^{\mathrm{n}} \mathrm{F}^{\mathrm{F}}\right) \geq \mathrm{cl}_{\mathrm{Y}}^{\mathrm{m}} \mathrm{f}(\mathrm{F})$.

6. QUOTIENT EXTENSIONS.

In this concluding section, we shall consider the circumstances under which $f_{\star}$ will possess certain quotient-type properties. We begin with definitions of the properties to be considered.

The term map will be used to mean a continuous, onto function. Note that if $f: X \rightarrow Y$ is onto, then any natural extension $f_{*}: X^{*} \rightarrow Y^{*}$ is also onto.

1. $\mathrm{f}$ is proper if $\mathrm{f}$ is a map and, whenever $\& \rightarrow \mathrm{y}$ in $\mathrm{Y}$, and $z$ is an u.f. on $x$ such that $f(\xi)=\&$, then there is $x \in f^{-1}(y)$ such that $₹ \rightarrow x$.

2. $\mathrm{f}$ is a convergence quotient map if $\mathrm{f}$ is a map and, whenever $\& \rightarrow \mathrm{y}$ in $\mathrm{Y}$, there is $\mathrm{x} \in \mathrm{f}^{-1}(\mathrm{y})$ and $\vec{x} \rightarrow \mathrm{x}$ in $\mathrm{x}$ such that $\mathrm{f}(\mathrm{F})=\&$.

3. $f$ is perfect if $f$ is a proper convergence quotient map.

4. $f$ is open if $f$ is a map and whenever \& is an u.f. on $Y$ which converges to $y$, and $x \in f^{-1}(y)$, then there is an u.f. $y^{\rightarrow} x$ such that $f(x)=\&$.

5. $f$ is closure-preserving if $A \subseteq X$ implies $f\left(c 1_{X} A\right)=c 1_{Y} f(A)$.

Further information about these properties may be found in [6], [8], and

[10]. Recall that if $P$ represents any of the above properties, then $f: X \rightarrow Y$ is said to have property $\underline{\theta-P}$ if $\mathrm{f}: \mathrm{r}_{1} \mathrm{X} \rightarrow \mathrm{r}_{1} \mathrm{Y}$ has property $\mathrm{P}$. 
It is clear that a proper map is weakly proper, and that a weakly proper map onto a $\mathrm{T}_{2}$ space is proper.

In all of the propositions that follow, $f$ denotes a function from a space $X$ into a space $Y$, and $f_{\star}: X^{*} \rightarrow Y^{*}$ denotes a natural extension of $f$.

PROPOSITION 6.1. If $f$ is a proper map, then $f_{*}$ is also a proper map.

PROOF. Suppose that $G$ is an u.f. on $X^{*}$ such that $f_{\star}(G) \rightarrow \alpha$ in $Y^{*}$. Then there exists an u.f. $K$ on $Y$ such that $f_{*}(G) \geq K^{*}$, where $K^{*} \rightarrow \alpha$ in $\mathrm{Y}^{*}$. Suppose that $\mathrm{G} \rightarrow \beta$ in $\mathrm{X}^{*}$; then there exists a filter \& on $\mathrm{X}$ such that $\& \rightarrow \beta$ in $x^{*}$ and $a \geq \xi^{*}$. Hence $f_{*}(a) \geq f_{*}\left(\xi^{*}\right)=f(\xi)^{*}$ since $f$ is a proper map, and thus $\mathrm{f}(\&)^{*}$ and $K^{*}$ are not disjoint filters on $\mathrm{Y}^{*}$. This implies that $f(\&)$ and $K$ are not disjoint filters on $Y$; consequently, $f(\&)=K$.

If $\alpha \notin Y$, then it follows that $f_{*}(\beta)=\alpha$. If $\alpha \in Y$, then since $f$ is a proper map, $\& \rightarrow x$ in $x$ for some $x \in f^{-1}(\alpha)$, and thus $G \rightarrow x$ in $x^{*}$. It follows that $f_{*}$ is a proper map.

PROPOSITION 6.2. If $\mathrm{f}$ is a convergence quotient map, then $f_{\star}$ is a convergence quotient map iff $f_{*}$ is continuous.

PROOF. The relation $f(A)^{*} \subseteq f_{*}\left(A^{*}\right)$ is satisfied for each subset $A$ of $X$, and hence $f_{\star}\left(z^{*}\right) \leq f(z)$ for each filter $z$ on $X$. Suppose that $\& * \rightarrow \alpha$ in $Y^{*}$ and $\alpha \notin Y$; let $\sharp$ be any u.f. on $X$ such that $f(\xi)=\&$. Then $f_{\star}(\sharp)=\alpha$ and

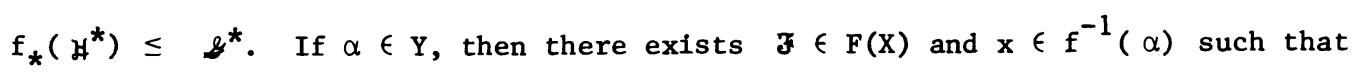
$\exists \rightarrow x$ in $x$ and $f(F)=\&$, since $f$ is a convergence quotient map. Since $f_{*}\left(z^{*}\right) \leq \xi^{*}$, it follows that $f_{*}$ is a convergence quotient map precisely when $f_{\star}$ is a continuous map.

COROLLARY 6.3. $f_{*}$ is a perfect map whenever $f$ is a perfect map.

PROPOSITION 6.4. If $\mathrm{f}$ is an open, proper map, then $f_{*}$ is open, proper, $\theta$-open, and $\theta$-proper. 
PROOF. It follows by Proposition 6.1 that $f_{*}$ is proper. If $f_{*}$ is also open, then it follows from Theorem 4.2, [14], that $f_{\star}$ is also $\sigma$-open and $\sigma$-proper. Thus it remains only to show that $f_{*}$ is open.

Let $G \in U\left(Y^{*}\right)$ such that $G \rightarrow \alpha$ in $Y^{*}$, and let $\beta \in f_{*}^{-1}(\alpha)$. Then there is $\& \in U(Y)$ such that $a \geq \&^{*}$ and $z^{*} \rightarrow \alpha$ in $Y^{*}$. If $\alpha \notin Y$, then $\beta \notin X$, and hence there exists exactly one u.f. $\quad$ on such that $\rightarrow \beta$ in $x^{*}$. Thus $f(z)=\&$, and since $f$ is a proper map, $f_{*}\left(z^{*}\right)=\&^{*} \leq G$. Let $B \in U\left(X^{*}\right)$ contain both $z^{*}$ and $f_{*}^{-1}(G)$; then $f_{*}(\beta)=G$, and $B \rightarrow B$ in $x^{*}$.

If $\alpha \in \mathrm{Y}$ then, since $\mathrm{f}$ is a proper map, $\beta \in \mathrm{X}$, and since $\mathrm{f}$ is an open map, there is $\exists \in U(X)$ such that $\rightarrow \beta$ in $X$ and $f(z)=\&$. Then, as in the argument of the preceding paragraph, there exists an u.f. $B \rightarrow B$ in $x^{*}$ such that $f_{*}(B)=Q$ this establishes that $f_{\star}$ is an open map.

We omit the straightforward proof the next proposition.

PROPOSITION 6.5. If $f$ is perfect $\theta$-proper map, then $f_{*}$ is a $\theta$-perfect map.

Propositions 6.4 and 6.5 yield the following corollary.

COROLLARY 6.6. If $\mathrm{f}$ is an open perfect map, then $f_{\star}$ is a $\theta$-perfect map.

PROPOSITION 6.7. If $\mathrm{f}$ is a convergent quotient map which is closure preserving, then $f_{\star}$ is a $\theta$-convergence quotient map.

PROOF. Suppose that $\mathrm{a} \rightarrow \alpha$ in $\mathrm{r}_{1} \mathrm{Y}^{*}$. Then there is $\& \in \mathrm{F}(\mathrm{Y})$ such that $\&^{*} \rightarrow \alpha$ and $a \geq \mathrm{cl}_{\mathrm{Y}^{*}}^{\mathrm{n}} \&$. If $\alpha \notin \mathrm{Y}$, then it may be assumed that $\alpha=\& \in \mathrm{Y}^{\prime}$. Let $z \in U(X)$ such that $f(z)=\&$; then $\beta=\widetilde{F} \in X^{\prime}$ and $f_{*}(\beta)=\alpha$. By Lemma 2.4 and the assumption that $f$ is closure-preserving, it follows that $f_{*}\left(c 1_{X^{*}}^{n+1} 3^{*}\right) \leq f_{*}\left(\left(c 1_{X}^{n}\right)^{*}\right) \leq c 1_{Y}^{*} f\left(c 1_{X}^{n} z^{*}\right)=c 1_{Y}^{*} c 1_{Y}^{n} f(z) \leq c 1_{Y^{*}}^{n} \xi^{*}$. If $\alpha \in \mathrm{Y}$ then, since $\mathrm{f}$ is a convergence quotient map, there is $3 \rightarrow \beta \in \mathrm{f}^{-1}(\alpha)$ such that $f(z)=\&$. Again, $f_{*}\left(\mathrm{cl}_{\mathrm{X}^{*}}^{\mathrm{n}+1} \xi\right) \leq \mathrm{cl}_{\mathrm{y}^{*}}^{\mathrm{n}} \xi^{*}$. In both cases $\mathrm{cl}_{\mathrm{X}^{*}}^{\mathrm{n}+1} z^{*} \rightarrow \beta$ in $r_{1} x^{*}$. If $K=f_{*}^{-1}(a) \vee c 1_{x^{*}}^{n+1} z$, then $K \rightarrow \beta$ in $r_{1} x^{*}$ and $f_{*}(K)=a$. Thus $f_{*}$ is a $\theta$-convergence quotient map. 
The final proposition follows immediately from Proposition 5.6.

PROPOSITION 6.8. If $\mathrm{f}$ is $\theta$-continuous and closure preserving, then $f_{*}$ is $\theta$-continuous.

We conclude by citing, without detail, some examples which place limitations on the types of results obtained in this section. The function $f$ constructed in Example 4.3 of [14] is perfect but not $\theta$-proper; it is also not difficult to show that in this case $f_{\star}$ is not $\theta$-proper. Thus, in Corollary 6.6 , one cannot drop the assumption that $f$ is open. There are other examples which show that $f_{*}$ may fail to be continuous when $f$ is an open, convergence quotient map, and that $f_{\star}$ may $f a i l$ to be open when $f$ is open and $f_{*}$ is continuous.

\section{REFERENCES}

[1] Binz, E., "Continuous Convergence in C(X)," Lecture Notes in Mathematics. Berlin, Heidelberg, New York: Springer-Verlag, Vol. 469 (1975).

[2] Dickman, R.F. and J.R. Porter. " $\theta$-Perfect and $\theta$-absolutely Closed Functions," Illinois, J. Math 21 (1977) 42-60.

[3] Fomin, S., "Extensions of Topological Spaces," Ann. Math. 44 (1943) 471-480.

[4] Gazik, R.J., "Regularity of Richardson's Compactification," Can. J. Math. 26 (1974) 1289-1293.

[5] Herrmann, R.A., "Perfect Maps on Convergence Spaces," Bull. Austral. Math. Soc. (To appear).

[6] Kent, D.C., "Convergence Quotient Maps," Fund. Math. 65 (1969) 197-205.

[7] Kent, D.C. and G.D. Richardson, "Compactifications of Convergence Spaces," Internat. J. Math. and Math. Sci. 2 (1979) 345-368.

[8] "Open and Proper Maps Between Convergence Spaces," Czech. Math. J. 23 (1973) 15-23.

[9] _Completely Regular and $\omega$-Regular Spaces," Proc. Conference on Conv.'Structures, Cameron Univ., Lawton, OK., April 1980, 84-90.

[10] Lowen-Colebunders, Eva, "Open and Proper Maps Characterized by Continuous Setvalued Maps," Can. J. Math. (To appear). 
[11] Richardson, G.D., "A Stone-Čech Compactification for Limit Spaces," Proc. Amer. Math. Soc. 25 (1970) 403-404.

[12] Richardson, G.D. and D.C. Kent, "Regular Compactifications of Convergence Spaces," Proc. Amer. Math. Soc. 31 (1972) 571-573.

[13] , "The Regularity Series of a Convergence Space," Bull. Austral. Math. Soc. 13 (1975) 21-44.

[14] "The Regularity Series of a Convergence Space II," Bull. Austral. Math. Soc. 15(1976) 223-244. 


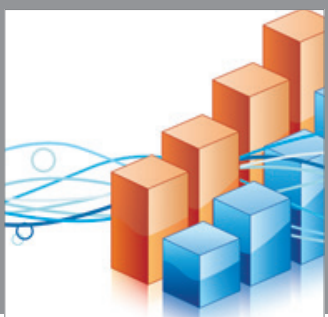

Advances in

Operations Research

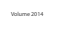

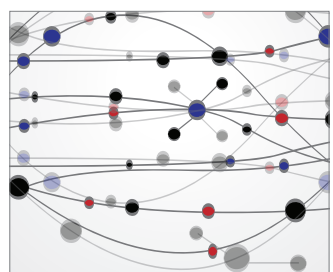

\section{The Scientific} World Journal
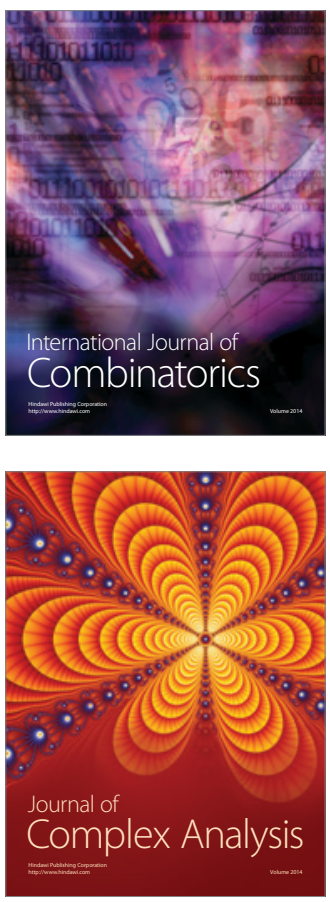

International Journal of

Mathematics and

Mathematical

Sciences
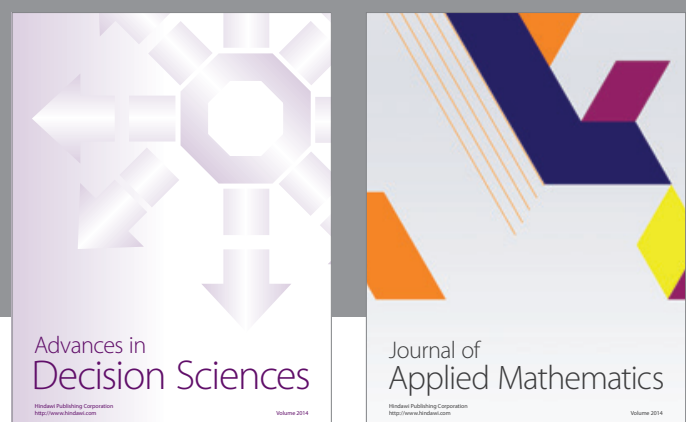

Journal of

Applied Mathematics
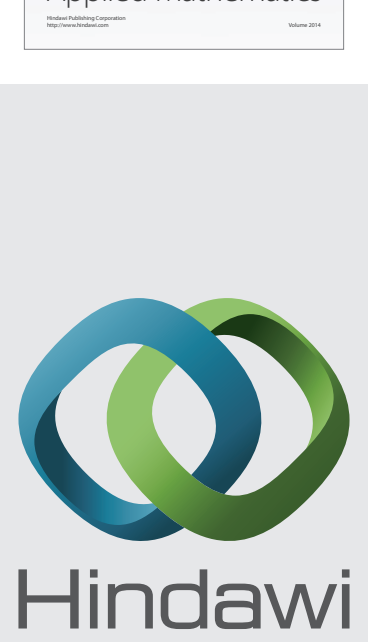

Submit your manuscripts at http://www.hindawi.com
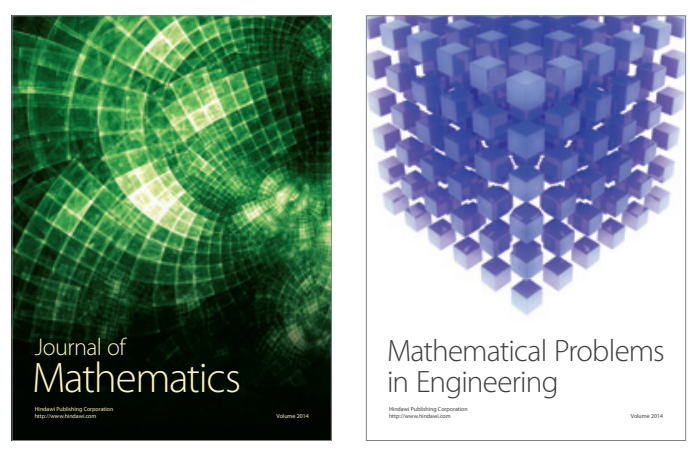

Mathematical Problems in Engineering
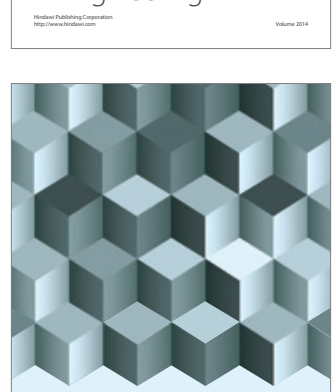

Journal of

Function Spaces
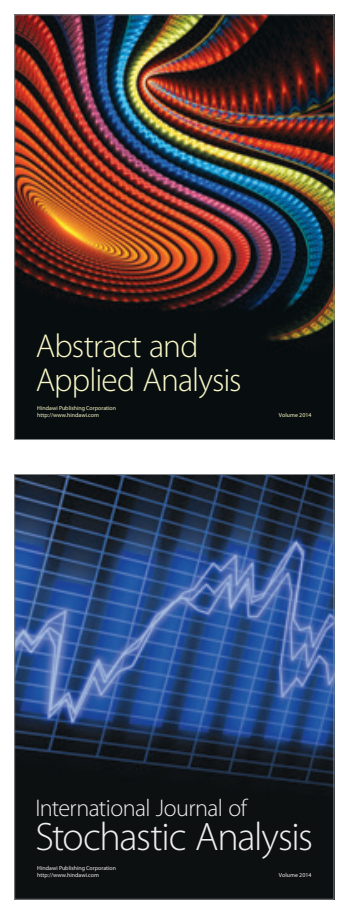

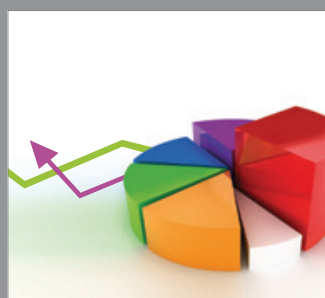

ournal of

Probability and Statistics

Promensencen
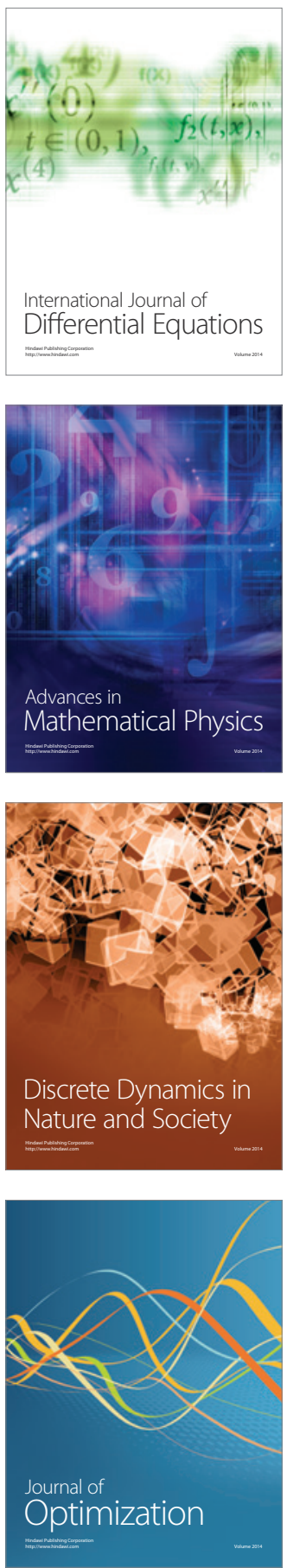